\title{
SUCCESSION OF MICROORGANISMS DURING COMPOSTING OF POULTRY MANURE
}

\author{
M. V. Myahka, S. M. Derkach, V. V. Volkohon, N. V. Lutsenko
}

Over the last decade poultry farming in Ukraine gained rapid development. According to the State Statistics Service of Ukraine, from 1998 to 2013 poultry population increased from 123.3 to 245.5 million units. Active development of the industry caused significant accumulation of waste products, including poultry manure - at the level of $1.5 \mathrm{mln}$ tons per year [14].

Part of manure is used to make fertilizers, but most enterprises accumulate that waste in settlers or storage clamps, accompanied by the development of pathogenic microbiota, and are unacceptable from the sanitary point of view. In addition, at such manure storage, significant loss of nitrogen is observed, which causes air and soil pollution, breaking the balance in ecosystems. That's why uncontrolled accumulation of poultry waste is an urgent problem for almost all regions of Ukraine [12], which soon could lead to environmental disaster.

There is a number of technologies for processing poultry manure that differ in the nature of fermentation of organic matter, use of supplements, duration of production processes, quality of output products $[6 ; 7$; 10]. However, these technologies are multistage and energy-intensive and do not allow to get bio-organic fertilizers of high quality, because they do not take into account the peculiarities of microbial processes that occur during composting. Some scientific studies involve the use of bacterial cultures in composting, but limit it to mechanical introduction of bacterial suspension to the substrate without learning the peculiarities of colonization rate of microorganisms and their development $[8 ; 9 ; 11]$. Meanwhile, the quality and safety of the final product in most cases is determined by the dominant microbiota. Therefore, in our view, solving the problem of manure disposal is possible by controlling the state of microbial coenosis of the substrate during composting. Perspective may be the improvement of product by the introduction of microorganisms to the substrate that are able not only to speed up the composting process, but also to ensure the accumulation of biologically active substances, that can then have a positive impact on the growth and development of plants.

In connection with the above, the goal of our work was to determine the features of successions of microorganisms during composting of chicken manure.

Materials and methods. Composting provided preset setting of optimal C:N ratio in the substrate at the rate of $20: 1$, as chicken manure has narrow ratio of 9.6:1. Its optimization was performed by mixing the manure with straw and/or peat as the source of carbon, in calculated quantities.

Plastic containers were used in the experiment, where studied substrates based on manure were placed. To optimize the ratio of $\mathrm{C}: \mathrm{N}$ to the level of $20: 1$ according to experiment variants, chopped straw and peat (mixture number 1 ) or only peat (mix number 2) were added to the manure in calculated amounts. Repetition of the experiment quadruple.

Accordingly, the experiment design included the following variants:

1 - control (chicken manure) with the ratio of $\mathrm{C}: \mathrm{N}=9.6: 1$;

2 - compost mixture number 1 (chicken manure with straw and peat) with the ratio of $\mathrm{C}: \mathrm{N}=20: 1$;

3 - compost mixture number 2 (chicken manure with peat) with the ratio of $\mathrm{C}: \mathrm{N}=20: 1$.

Substrate humidity was maintained at the level of $70-75 \%$, stirring it once every two weeks.

During the composting changes in the number of representatives of particular environmental and trophic groups of microorganisms were studied: ammonifying bacteria - on meat-and-peptone agar (MPA), microorganisms absorbing mineral forms of nitrogen - on starch-and-ammonia agar (SAA), micromycetes - on wort-agar (WA) [13]. The account of the number of nitrogen fixation microorganisms was performed by critical dilution method using acetylene test 
on semisolid Ashby medium [4]. The number of microorganisms that dissolve difficult-toaccess phosphorus compounds was determined on Muromtsev medium [5]. The number of cellulose-fermenting bacteria was studied on liquid Imshenetskyi and Solntseva medium [3].

The content of carbon and nitrogen in composts was studied in dynamics by Anstet method modified by Ponomariova and Nikolaieva [1].

Statistical processing of the results was conducted by Dospiekhov [2].

Based on the obtained data, we can draw some conclusions:

1. The optimization of $\mathrm{C}: \mathrm{N}$ ratio at the level of 20:1 by adding straw and/or peat to chicken manure provides optimal conditions for the development of compost microbiota and performing mineralization processes.

2. Composting of manure without setting $\mathrm{C}: \mathrm{N}$ ratio optimization ensures the development of the representatives of the majority of studied groups of microorganisms only at the initial stages of composting. These results indicate a low level of mineralization of organic matter. This is the basis for the conclusion that it is inappropriate to compost pure chicken manure because under these conditions the process is carried out for too long.

3. In composts with optimized $\mathrm{C}: \mathrm{N}$ ratio the results of the studies of succession of microorganisms testify to gradual decomposition of complex organic compounds by some microorganisms and creating nutrient sources for others.

4. In composted mixtures with optimized carbon to nitrogen ratio two periods of active growth of microorganisms (month 4 and 6-7 of composting) and their decline (month 5 and 8 of composting) are observed.

Taking into account the features of dynamics of the development of microorganisms allows to ground favourable conditions and corresponding optimal period for the introduction of agronomically valuable microorganisms to composted mixtures. For example, the introduction of ammonifiers will be most effective in the early stages of composting, and diazotrophs - in the final. This will allow to enrich the composts with useful microbiota and get an innovative product of new quality. 